\title{
ON THE VOLUME IN HOMOGENEOUS SPACES
}

\section{MINORU KURITA}

Guldin-Pappus's theorem about the volume of a solid of rotation in the euclidean space has been generalized in two ways. G. Koenigs [1] and J. Hadamard [2] proved that the volume generated by a 1-parametric motion of a surface $D$ bounded by a closed curve $c$ is equal to $\sum_{i} a_{i} b_{i}+\sum_{i<j} a_{i j} b_{i j}$, where $a_{i}, a_{i j}=-a_{j i}(i, j=1,2,3)$ are quantities attached to $D$ with respect to a rectangular coordinate system, while $b_{j}, b_{i j}=-b_{j i}(i, j=1,2,3)$ are quantities determined by our motion. It is remarkable that $a_{i}, a_{i j}$ depend only on $c$ and not on $D$. The theorem was extended to the case of dimension $n$ by G. Guillaumin [3]. Another extension of Guldin-Pappus's theorem was obtained by the author [7] in the following way. The volume $V$ of a solid $B$ in the euclidean space of dimension $n$ is given by $\int v d \sigma$, where $v$ is an $(n-1)$-dimensional volume of a section of $B$ by one of the 1-parametric set of hyperplanes and $d \sigma$ is a component, orthogonal to the hyperplanes, of an arcelement of the locus of the center of gravitation of the section. An analogous result was obtained for the spherical space. In the present paper the author generalizes these results to the case of homogeneous spaces by the method of moving frames of E. Cartan and applies the results to various spaces, and states formulas of the integral geometry in the homogeneous spaces.

\section{Preliminaries}

1. In the first we quote the matters necessary for our purpose from [4] and [6]. Let $G$ be a group which operates on a space $M$ effectively and transitively. We take an element $p_{0}$ of $M$ and a set $H$ of all elements of $G$ which fix $p_{0}$. Any element $p$ of $M$ corresponds to a set $\sigma H(\sigma \in G)$ of $G / H$ and $M$ can be identified with $G / H$ in natural way. Now we take a set of elements upon which $G$ operates simply transitively and call each element of the set a frame of $M$, and to a point $p$ corresponds a set of frames $\sigma H R$, where $R$ is a

Received February 4, 1959. 
fixed frame. A relative displacement from a frame $T R$ to $S R(S, T \in G)$ is defined by $T^{-1} S$ and an absolute displacement by $S T^{-1}$.

2. Now we assume that $G$ is a Lie group of dimension $r$ and $H$ its closed connected subgroup of dimension $n-r$, and consider the homogeneous space $G / H$ which we call $M$. We denote by $S_{a}$ an element of $G$ with local parameters $a=\left(a_{1}, \ldots, a_{r}\right)$. Left invariant differential 1 -forms $\omega_{p}=\omega_{p}(a, d a) \quad(p=1$, $\ldots, r)$, called relative components, are obtained by taking linear parts in $d a=\left(d a_{1}, \ldots, d a_{r}\right)$ of the parameters $b=\left(b_{1}, \ldots, b_{r}\right)$ of $S_{b}=S_{a}^{-1} S_{a+d a}$, which is a relative displacement from $S_{a} R$ to $S_{a+d a} R$. Relative components $\omega_{p}=\omega_{p}(a, d a)$ are base of a vector space $V$ dual to the tangent space of $G$. By taking a suitable linear combination of $\omega_{p}$ with constant coefficients and denoting them $\omega_{p}$ anew we can assume $\omega_{i}(a, d a)=0(i=1, \ldots, n)$ for the elements $S_{a}, S_{a+d a}$ of $H$. These $\omega_{i}$ 's are called principal relative components of $M$. We can take suitable local parameters $x_{1}, \ldots, x_{n}, u_{n+1}, \ldots, u_{r}$, such that $x=\left(x_{1}, \ldots, x_{n}\right)$ are considered as local coordinates of $M$ and principal relative components are represented as

$$
\omega_{i}=\sum_{j} a_{i j}\left(x_{1}, \ldots, x_{n}, u_{n+1}, \ldots, u_{r}\right) d x_{j} . \quad(i, j=1, \ldots, n)
$$

Hereafter we take the indices in the following manner:

$$
\begin{aligned}
& p, q, s, u=1, \ldots, r \\
& i, j, k=1, \ldots, n \quad \alpha, \beta, r=n+1, \ldots, r .
\end{aligned}
$$

Then we have

$$
d \omega_{p}=\sum_{q s} \frac{1}{2} c_{q s p}\left[\omega_{q} \omega_{s}\right], \quad \text { where } \quad c_{q s p}=-c_{s q p}
$$

especially, $\quad d \omega_{i}=\sum_{j k} \frac{1}{2} c_{j k i}\left[\omega_{j} \omega_{k}\right]+\sum_{\alpha k} c_{\alpha k i}\left[\omega_{\alpha} \omega_{k}\right]$.

When we take frames $S_{a} S_{t} R$ and $S_{a+d a} S_{t+d t} R$ instead of $S_{a} R$ and $S_{a+d a} R$,

$$
\left(S_{a} S_{t}\right)^{-1}\left(S_{a+d a} S_{t+d t}\right)=S_{t}^{-1}\left(S_{a}^{-1} S_{a+d a}\right) S_{t} \cdot S_{t}^{-1} S_{t+d t}
$$

and denoting the relative components of $\left(S_{a} S_{t}\right)^{-1}\left(S_{a+d a} S_{t+d t}\right)$ by $\pi_{p}$ we have

$$
\pi_{p}=\sum_{q} t_{p q} \omega_{q}(a, d a)+\omega_{p}(t, d t)
$$

where $\left(t_{p q}\right)$ is a matrix representing an element of a linear adjoint group of $G$ corresponding to $S_{t}$. We have

$$
d t_{p q}=\sum_{u s} c_{u s p} \omega_{u l}(t, d t) t_{s q}
$$


If $S_{t}, S_{t+d t}$ belong to the connected subgroup $H$ of $G$, we have

and by (1.4)

$$
\begin{aligned}
& \omega_{i}(t, d t)=0 \\
& \pi_{i}=\sum_{j} t_{i j} \omega_{j}
\end{aligned}
$$

and as a special case of (1.5)

and

$$
d t_{i j}=\sum_{\alpha k} c_{\alpha k i} \omega_{\alpha}(t, d t) t_{k j}
$$

$$
t_{i \alpha}=0 \text {. }
$$

$\left(t_{i j}\right)$ is a matrix representing an element of a linear group of isotropy.

\section{Main theorems}

1. An invariant volume can be defined in $M=G / H$ when and only when the linear group of isotropy is unimodular, namely

$$
\operatorname{det}\left(t_{i j}\right)=1 \quad \text { for } \quad S_{t} \in H,
$$

in another words,

$$
\sum_{i} c_{a i i}=0,
$$

as we see by (1.7) and (1.8) (we have assumed that $H$ is connected). The volume element $d V$ is an exterior product of principal relative components

$$
d V=\left[\omega_{1} \omega_{2} \cdots \omega_{n}\right]
$$

with disregard to a constant multiplier.

Now we define an algebraic volume in $M$. Let $E_{n}$ be a euclidean space of dimension $n$ and $K$ be a measurable set of $E_{n}$. Let a differentiable mapping of $K$ into $M$ be $\varphi$, then a mapping $\varphi^{*}$ of differential forms in $M$ into forms in $K$ is naturally defined. We define $\int_{\varphi(K)}\left[\omega_{1} \omega_{2} \cdots \omega_{n}\right]$ by $\int_{K} \varphi^{*}\left[\omega_{1} \omega_{2} \cdots \omega_{n}\right]$ and call it an algebraic volume of $(\varphi, K)$ in $M$. We define an equivalence relation between $(\varphi, K)$ and $\left(\varphi^{\prime}, K^{\prime}\right)$ by the existence of a differentiable homeomorphism $f$ such that

$$
K^{\prime}=f(\dot{K}), \quad \varphi^{\prime}=\varphi f^{-1}
$$

An algebraic volume is a quantity defined for the equivalence class.

2. We take cartesian coordinates $x_{1}, \ldots, x_{n-1}, u$ in $E_{n}$ and consider a domain $D$ on a hyperplane defined by $u=0$. Let $\psi$ be a $1-1$ mapping of $D$ into $M$ which is univalent on tangent spaces. $\psi(D)$ is then an $n-1$ dimensional submanifold, namely a hypersurface. By a 1-parametric motion represented by 
$S_{t}(\in G, t=t(u))$ for an interval $I(0 \leqq u \leqq 1) \psi(D)$ generates a set of $M$. Putting

$$
\begin{aligned}
& K=D \times I \\
& \varphi(p)=S_{t}(\phi(x)) \text { for } p=\left(x_{1}, \ldots, x_{n-1}, u\right) \text { such that } \\
& \qquad x=\left(x_{1}, \ldots, x_{n-1}, 0\right) \in D
\end{aligned}
$$

an algebraic volume can be defined for $(\varphi, K)$. When we take an $n-1$ parametric set of frames $S_{a} R$ attached to each point $\psi(x)$ of $\psi(D), S_{t} S_{a} R$ is attached to a point $S_{t}(\psi(x))$. Let the relative components of

be

$$
\left(S_{t} S_{a}\right)^{-1}\left(S_{t+d t} S_{a+d a}\right), \quad S_{t}^{-1} S_{t+d t}, \quad S_{a}^{-1} S_{a+d a}
$$

respectively, then by the relation

$$
\left(S_{t} S_{a}\right)^{-1}\left(S_{t+d t} S_{a+d a}\right)=S_{a}^{-1}\left(S_{t}^{-1} S_{t+d t}\right) S_{a} \cdot S_{a}^{-1} S_{a+d a}
$$

we have

$$
\pi_{i}=\omega_{i}+\sum_{p} a_{i p} \tau_{p}
$$

where $\left(a_{q p}\right)$ is an element of a linear adjoint group corresponding to $S_{a}$.

As a volume element $d V$ of $(\varphi, K)$ we have

$$
d V=\left[\pi_{1} \pi_{2} \cdots \pi_{n}\right]
$$

and by (2.4)

$$
\left[\pi_{1} \pi_{2} \cdots \pi_{n}\right]=\sum_{i p} a_{i p}\left[\omega_{1} \cdots \omega_{i-1} \tau_{p} \omega_{i+1} \cdots \omega_{n}\right],
$$

because the set of frames $S_{a} R$ is $n-1$-parametric and $S_{t}$ is 1-parametric. Putting

$$
\Omega_{p}=\sum_{i}(-1)^{i-1} a_{i p}\left[\omega_{1} \cdots \hat{\omega}_{i} \cdots \omega_{n}\right],
$$

where $\hat{\omega}_{i}$ means that a term $\omega_{i}$ does not appear in the product, we have

$$
d V=\sum_{\nu}\left[\tau_{p} \Omega_{p}\right]
$$

We call an algebraic volume $V$ of $(\varphi, K)$ a volume generated by a hypersurface $\psi(D)$ under a 1 -parametric motion $S_{t}(t=t(u), 0 \leqq u \leqq 1)$. Putting

$$
X_{p}=(-1)^{n-1} \int_{\psi(D)} \Omega_{p}, \quad Y_{p}=\int_{0 \leqq u \leqq 1} \tau_{p}
$$

we get the following theorem by integrating (2.6).

TheOREM 1. An algebraic volume $V$ generated by a 1-parametric motion of a hypersurface $\psi(D)$ is given by 


$$
V=\sum_{p} X_{p} Y_{p}
$$

where $X_{p}$ 's are quantities determined by $\psi(D)$ and $Y_{p}$ 's are those determined by our motion.

This is a generalization of the theorem of Koenigs-Hadamard.

3. We consider $\Omega_{p}$ in detail. Until now $S_{a} R$ has been attached to a point of $\psi(D)$, and $S_{a}$ has been of $n-1$-parameters. Now we consider $S_{a}$ which is $r$-parametric, and construct forms

$$
\Omega_{p}=\sum_{i}(-1)^{i-1} a_{i p}\left[\omega_{1} \cdots \hat{\omega}_{i} \cdots \omega_{n}\right],
$$

where $\omega_{t}$ 's are principal relative components of $M$ corresponding to $S_{a}^{-1} S_{a+d a}$ and $\left(a_{q p}\right)$ is an element of a linear adjoint group corresponding to $S_{a}$. Then we get

TheOREM 2. Differential forms $\Omega_{p}$ are closed and are forms on $M$.

Verification runs as follows by the use of (1.5) (1.2):

$$
\begin{aligned}
d \Omega_{p}= & \sum_{i}(-1)^{i-1}\left[d a_{i p} \omega_{1} \cdots \hat{\omega}_{i} \cdots \omega_{n}\right] \\
& +\sum_{i>j}(-1)^{i+j} a_{i p}\left[\omega_{1} \cdots d \omega_{j} \cdots \hat{\omega}_{i} \cdots \omega_{n}\right] \\
& +\sum_{i<j}(-1)^{i+j-1} a_{i p}\left[\omega_{1} \cdots \hat{\omega}_{i} \cdots d \omega_{j} \cdots \omega_{n}\right] \\
= & \sum_{\alpha k i}(-1)^{i-1} c_{\alpha k i} a_{k p}\left[\omega_{\alpha} \omega_{1} \cdots \hat{\omega}_{i} \cdots \omega_{n}\right] \\
& +\sum_{h k i}(-1)^{i-1} c_{h k i} a_{k p}\left[\omega_{h} \omega_{1} \cdots \hat{\omega}_{i} \cdots \omega_{n}\right] \\
& +\sum_{\alpha h i}(-1)^{i-1} c_{h \alpha i} a_{\alpha p}\left[\omega_{h} \omega_{1} \cdots \hat{\omega}_{i} \cdots \omega_{n}\right] \\
& +\sum_{\alpha, i>j, k}(-1)^{i+j} c_{\alpha k j} a_{i p}\left[\omega_{1} \cdots \omega_{j-1} \omega_{\alpha} \omega_{k} \omega_{j+1} \cdots \hat{\omega}_{i} \cdots \omega_{n}\right] \\
& +\sum_{\alpha, i<j, k}(-1)^{i+j-1} c_{\alpha k j} a_{i p}\left[\omega_{1} \cdots \hat{\omega}_{i} \cdots \omega_{j-1} \omega_{\alpha} \omega_{k} \omega_{j+1} \cdots \omega_{n}\right] \\
& +\sum_{i>j, h, k}(-1)^{i+j} \frac{1}{2} c_{h k j} a_{i p}\left[\omega_{1} \cdots \omega_{j-1} \omega_{h} \omega_{k} \omega_{j+1} \cdots \hat{\omega}_{i} \cdots \omega_{n}\right] \\
& +\sum_{i<j, h, k}(-1)^{i+j-1} \frac{1}{2} c_{h k j} a_{i p}\left[\omega_{1} \cdots \hat{\omega}_{i} \cdots \omega_{j-1} \omega_{h} \omega_{k} \omega_{j+1} \cdots \omega_{n}\right] \\
= & \sum_{\alpha i j}(-1)^{j-1} c_{\alpha i j} a_{i p}\left[\omega_{\alpha} \omega_{1} \cdots \hat{\omega}_{j} \cdots \omega_{n}\right] \\
& +\sum_{i j} c_{j i j} a_{i p}\left[\omega_{1} \cdots \omega_{n}\right]+\sum_{\alpha i} c_{i \alpha i} a_{\alpha p}\left[\omega_{1} \cdots \omega_{n}\right] \\
& +\sum_{\alpha, i>j}(-1)^{i-1} c_{\alpha j j} a_{i p}\left[\omega_{\alpha} \omega_{1} \cdots \hat{\omega}_{i} \cdots \omega_{n}\right] \\
& +\sum_{\alpha, i>j}(-1)^{j} c_{\alpha i j} a_{i p}\left[\omega_{\alpha} \omega_{1} \cdots \hat{\omega}_{j} \cdots \omega_{n}\right] \\
& +\sum_{\alpha, i<j}(-1)^{i} c_{\alpha j j} a_{i p}\left[\omega_{\alpha} \omega_{1} \cdots \hat{\omega}_{i} \cdots \omega_{n}\right]
\end{aligned}
$$




$$
\begin{aligned}
& +\sum_{\alpha, i<j}(-1)^{j} c_{\alpha i j} a_{i p}\left[\omega_{\alpha} \omega_{1} \cdots \hat{\omega}_{j} \cdots \omega_{n}\right] \\
& -\sum_{i>j} c_{j i j} a_{i p}\left[\omega_{1} \cdots \omega_{n}\right]-\sum_{i<j} c_{j i j} a_{i p}\left[\omega_{1} \cdots \omega_{n}\right] \\
= & \sum_{\alpha}\left(\sum_{j} c_{\alpha j j}\right)\left(\sum_{i}(-1)^{i-1} a_{i p}\left[\omega_{\alpha} \omega_{1} \cdots \hat{\omega}_{i} \cdots \omega_{n}\right]+a_{\alpha p}\left[\omega_{1} \cdots \omega_{n}\right]\right)
\end{aligned}
$$

and so by virtue of (2.2)

$$
d \Omega_{p}=0 .
$$

When we take local parameters $x_{1}, \ldots, x_{n}, u_{n+1}, \ldots, u_{r}$, for which (1.1) holds good, we have

$$
\Omega_{p}=\sum_{i} b_{i p}(x, u)\left[d x_{1} \cdots \hat{d x}_{i} \cdots d x_{n}\right]
$$

and by virtue of $(2.10) b_{i p}(x, u)$ do not contain $u$, which proves $\Omega_{p}$ are forms on $M=G / H$.

$\Omega_{p}$ are forms on $M$, but they depend on $S_{a}$ and are not invariant for displacement. They are not intrinsic in this sense. In a simply connected domain in $M$ there exist forms $\Pi_{p}$ such that $\Omega_{p}=d \Pi_{p}$. If the domain $D$ has regular boundary $C$ and $\psi(D)$ is in the domain above stated, we get

$$
X_{p}=(-1)^{n-1} \int_{\psi(D)} \Omega_{p}=\int_{\psi(D)} d \Pi_{p}=\int_{\psi(C)} \Pi_{p}
$$

and $X_{p}$ depend on $(\psi, C)$ and not on $(\psi, D)$.

4. If we take $S_{c}(\psi(D))$ in stead of $\psi(D)$ with $S_{c}$ constant in $G$, frames $\bar{S}_{a} R=S_{c} S_{a} R$ are attached to it, and if we take $\bar{S}_{t}$ such that

$\bar{S}_{t}=S_{t} S_{c}^{-1}$,
we have
$\bar{S}_{t} \bar{S}_{a}=S_{t} S_{a}$,

and the motion of $\psi(D)$ by $S_{t}$ is the same with that of $S_{c}(\psi(D))$ by $\bar{S}_{t}$. Owing to the relation

$$
\bar{S}_{a}^{-1}\left(S_{b}^{-1} S_{b+d b}\right) \bar{S}_{a}=S_{a}^{-1} S_{c}^{-1}\left(S_{b}^{-1} S_{b+d b}\right) S_{c} S_{a}
$$

an element of a linear adjoint group corresponding to $\bar{S}_{a}$ is a product of those of $S_{a}$ and $S_{t}$, and

$$
\bar{a}_{s p}=\sum_{q} a_{s q} c_{q p}
$$

where $\left(\bar{a}_{s p}\right),\left(a_{s q}\right),\left(c_{q p}\right)$ correspond to $\bar{S}_{a}, S_{a}, S_{c}$ respectively. By the relation $\bar{S}_{a}^{-1} \bar{S}_{a+d a}=S_{a}^{-1} S_{a+d a}$ we have $\bar{\omega}_{i}=\omega_{i}$, and putting 
we get

$$
\Omega_{p}=\sum_{i}(-1)^{i-1} \bar{a}_{i p}\left[\bar{\omega}_{1} \cdots \hat{\bar{\omega}}_{i} \cdots \bar{\omega}_{n}\right]
$$

and also for

$$
\bar{\Omega}_{p}=\sum_{q} \Omega_{q} c_{q p}
$$

$$
\begin{gathered}
X_{p}=(-1)^{n-1} \int \Omega_{p}, \quad \bar{X}_{p}=(-1)^{n-1} \int^{2} \bar{\Omega}_{p}, \\
\bar{X}_{p}=\sum_{q} X_{q} c_{q p} .
\end{gathered}
$$

By the use of (2.14) we can nomalize $X_{p}$, namely by taking $S_{c}$ suitably we reduce $X_{p}$ 's to a system which is as simple as possible. Examples will be given later.

5. Next we turn to another generalization of Guldin-Pappus's theorem. We assume $M=G / H$ has an $n$-1-dimensional submanifold $L$ which is transformed into itself transitively by a certain connected closed subgroup of $G$. We call $L$ a $W$-hypersurface. $L$ is derived from a solution of a set of completely integrable system

$$
\rho_{v}=0 \quad(v=1,2, \ldots, k)
$$

by E. Cartan's theory, where $\rho_{v}^{\prime}$ 's are linearly independent linear combinations of relative components with constant coefficients. As $L$ is of dimension $n-1$, principal relative components $\omega_{i}$ are not independent along $L$, and so by taking a suitable linear combination of them and suitable linear combinations of relative components $\omega_{p}$, both of constant coefficients, we can assume anew

$$
\omega_{1}=0, \quad \omega_{\lambda}=0 \quad(\lambda=n+1, \ldots, n+k-1)
$$

instead of (2.15). As this system is completely integrable we have

$$
\begin{array}{lll}
c_{i j 1}=0 & c_{i \sigma 1}=0 & c_{o p 1}=0 \\
c_{i j \lambda}=0 & c_{i \sigma \lambda}=0 & c_{\sigma p \lambda}=0,
\end{array}
$$

where

$$
\begin{aligned}
& i, j=2, \ldots, n \\
& \lambda=n+1, \ldots, n+k-1 \quad \sigma, \rho=n+k, 1, \ldots, r
\end{aligned}
$$

We take up a solution of (2.16) which contains an identity of $G$ and denote each element of the solution by $S_{a}$ which generates a subgroup $J$ of $G$. Then a set of frames $S_{a} R$ is attached to all points of a submanifold of $M$ which we can assume to be $L$. If $J$ is connected we have by virtue of (1.9)

$$
a_{1 p}=0, \quad a_{\lambda p}=0, \quad a_{1 i}=0, \quad a_{\lambda i}=0
$$

with the indices as in $(2.17)$, where $\left(a_{p q}\right)$ is an element of a linear adjoint 
group corresponding to $S_{a} \in J$. The forms (2.9) reduce to

$$
\Omega_{p}=a_{1 p}\left[\omega_{2} \cdots \omega_{n}\right]
$$

on the manifold $L$ and we get

$$
\begin{gathered}
\Omega_{i}=0, \quad \Omega_{\mathrm{p}}=0 \\
(i, j=2, \ldots, n, \quad \rho=n+k, \ldots, r)
\end{gathered}
$$

by virtue of $(2.18)$.

We consider a euclidean space of dimension $n$ with coordinates $x_{1}, \ldots$, $x_{n-1}, u$ and a 1 -parametric set of domains $D(u)(0 \leqq u \leqq 1)$ contained in a domain $D$ on the hyperplane $u=0$. Let $\psi$ be a differentiable mapping which maps $D$ on the $W$-hypersurface $L$, and $S_{\iota}(t=t(u), 0 \leqq u \leqq 1)$ be a 1-parametric motion on $M$. Then $S_{t}(\psi(D(u))$ generates a part of $M$ and a volume element $d V$ is given by

$$
d V=\sum_{p}\left[\tau_{p} \Omega_{p}\right]
$$

by taking an $n-1$-parametric set of frames $S_{a} R$ along $L$. If $K$ is a set of points $p=\left(x_{1}, \ldots, x_{n-1}, u\right)$ such that $x=\left(x_{1}, \ldots, x_{n-1}, 0\right) \in D(u)$ and $\varphi$ is defined by $\varphi(p)=S_{t}(\psi(x))$, an algebraic volume of $(\varphi, K)$ is given by

$$
V=\sum_{p} \int X_{p} \tau_{p}
$$

when we put

$$
X_{p}=X_{p}(u)=(-1)^{n-1} \int_{\psi(D(u))} \Omega_{p}
$$

By virtue of (2.19) we have $X_{i}=0, X_{p}=0$ and so

$$
V=\int X_{1} \tau_{1}+\sum_{\lambda} \int X_{\lambda} \tau_{\lambda} \quad(\lambda=n+1, \ldots, n+k-1) .
$$

Thus we get

TheOREM 3. An algebraic volume generated by a 1-parametric set of $W$ hypersurfaces is given by (2.23).

$X_{p}$ 's are quantities depending on $(\psi, C(u))$, where $C(u)$ is a boundary of $D(u)$.

We can simplify the formula $(2,23)$ in the following manner. If we take $\bar{S}_{a}$, and $\bar{S}_{t}$ such that 


$$
\bar{S}_{a}=S_{c} S_{a} \quad \bar{S}_{t}=S_{t} S_{c}^{-1}
$$

where $\dot{S}_{c}(c=c(u))$ belong to the subgroup $J$, we have

$$
\begin{gathered}
\bar{X}_{1}=X_{1} c_{11}+\sum_{\lambda} X_{\lambda} c_{\lambda 1}, \quad \bar{X}_{\lambda}=X_{1} c_{1 \lambda}+\sum_{\mu} X_{\mu} c_{\mu \lambda} . \\
(\lambda, \mu=n+1, \ldots, \quad n+k-1)
\end{gathered}
$$

By taking $c=c(u)$ suitably we can simplify $X_{p}$. Especially if the linear group defined by linear transformations (2.24), namely a linear group of isotropy on a homogeneous space $G / J$, is transitive, we can reduce in such a way that $\bar{X}_{\lambda}=0$ hold good, and we get from $(2.23)$

$$
V=\int X_{1} \tau_{1}
$$

Examples will be given in the following section.

\section{Applications}

We take in the euclidean space of dimension $n$ rectangular coordinates $x=\left(x_{1}, \ldots, x_{n}\right)$. A displacement from a point $\xi=\left(\xi_{1}, \ldots, \xi_{n}\right)$ to $\xi^{\prime}=\left(\xi_{1}^{\prime}\right.$, $\left.\ldots, \xi_{n}^{\prime}\right)$ is given by

$$
\xi \rightarrow \xi^{\prime}: \xi_{i}^{\prime}=\sum_{j} p_{j i} \xi_{j}+x_{i} \quad(i, j=1, \ldots, n),
$$

where $\left(p_{i j}\right)$ is a proper orthogomal matrix. Let the fundamental frame $R$ be given by a set of vectors

$$
A^{\circ}=(0, \ldots, 0), \quad e_{1}^{\circ}=(1,0, \ldots, 0), \ldots, e_{n}^{\circ}=(0, \ldots, 0,1)
$$

and a frame derived from (3.2) by the displacement (3.1) be

$$
A=\left(x_{1}, \ldots, x_{n}\right), \quad e_{1}=\left(p_{11}, \ldots, p_{1 n}\right), \ldots, e_{n}=\left(p_{n 1}, \ldots, p_{n n}\right) .
$$

When we put

$$
R^{\circ}=\left(\begin{array}{c}
A^{\circ} \\
e_{1}^{\circ} \\
\vdots \\
e_{n}^{\circ}
\end{array}\right), \quad R=\left(\begin{array}{c}
A \\
e_{1} \\
\vdots \\
e_{n}
\end{array}\right), \quad S=\left(\begin{array}{cccc}
1 & x_{1} & \cdots & x_{n} \\
0 & p_{11} & \cdots & p_{1 n} \\
\cdots & \cdots & \cdots & \cdots \\
0 & p_{n 1} & \cdots & p_{n n}
\end{array}\right),
$$

we get

$$
R=S R^{\circ} \text {. }
$$

We take another displacement represented by

$$
\ddot{\xi}^{\prime} \rightarrow \xi^{\prime \prime}: \xi_{i}^{\prime \prime}=\sum_{l} q_{j i} \xi_{j}^{\prime}+y_{i}
$$


and denote a corresponding frame transformation by

$$
R=T R^{\circ} \text {. }
$$

To the displacement $\xi \rightarrow \xi^{\prime \prime}$, which is a composition of (3.1) and (3.5), corresponds a frame transformation from $R^{\circ}$ to $R$ such that

$$
R=S T R^{\circ} \text {. }
$$

Relative displacement from $S R$ to $T R$ is given by $T S^{-1}$ and by virtue of

$$
(S+d S) S^{-1}=E+d S S^{-1}
$$

relative components $\omega_{i}, \omega_{i j}=-\omega_{j i}(i, j=1, \ldots, n)$ are coefficients of $d S S^{-1}$, namely

$$
d S S^{-1}=\left(\begin{array}{cccc}
0 & \omega_{1} & \cdots & \omega_{n} \\
0 & \omega_{11} & \cdots & \omega_{1 n} \\
\cdots & \cdots & \cdots & \cdots \\
0 & \omega_{n 1} & \cdots & \omega_{n n}
\end{array}\right)
$$

This is usually represented as

$$
d A=\sum_{i} \omega_{i} e_{i}, \quad d e_{i}=\sum_{j} \omega_{i j} e_{j} \quad(i, j=1, \ldots, n) .
$$

Next we take a fixed displacement represented by a matrix $C$. Relative components of the displacement from $C S R$ to $C(S+d S) R$ are given by the coefficients of a matrix

$$
d(C S)(C S)^{-1}=C\left(d S S^{-1}\right) C^{-1}
$$

We denote as

$$
C=\left(\begin{array}{ccccc}
1 & c_{1} & \cdots & c_{n} \\
0 & c_{11} & \cdots & c_{n n} \\
\cdots & \cdots & \cdots & \cdots \\
0 & c_{n 1} & \cdots & c_{n n}
\end{array}\right), \quad d(C S)(C S)^{-1}=\left(\begin{array}{cccc}
0 & \bar{\omega}_{1} & \cdots & \bar{\omega}_{n} \\
0 & \bar{\omega}_{11} & \cdots & \bar{\omega}_{1 n} \\
\cdots & \cdots & \cdots & \cdots \\
0 & \bar{\omega}_{n 1} & \cdots & \bar{\omega}_{n n}
\end{array}\right)
$$

with a proper orthogonal matrix $\left(c_{i j}\right)$, and we get

$$
\begin{aligned}
& \bar{\omega}_{i}=\sum_{k} c_{i k}\left(\omega_{k}+\sum_{j} c_{j} \omega_{j k}\right)=\sum_{k} c_{i k} \omega_{k}+\sum_{j<k}\left(c_{j} c_{i k}-c_{k} c_{i j}\right) \omega_{j k} \\
& \bar{\omega}_{i j}=\sum_{k h} c_{i k} c_{j h} \omega_{k h}=\sum_{k<h}\left(c_{i k} c_{j h}-c_{i h} c_{j k}\right) \omega_{k h} .
\end{aligned}
$$

This is a transformation of a linear adjoint group corresponding to $C$.

Let $S$ be as in (3.3) and $T$ be a matrix representing a 1-parametric motion and put 


$$
\begin{gathered}
d(S T)(S T)^{-1}=\left(\begin{array}{cccc}
0 & \pi_{1} & \cdots & \pi_{n} \\
0 & \pi_{11} & \cdots & \pi_{1 n} \\
\cdots & \cdots & \cdots & \cdots \\
0 & \pi_{n 1} & \cdots & \pi_{n n}
\end{array}\right), \quad d T T^{-1}=\left(\begin{array}{cccc}
0 & \tau_{1} & \cdots & \tau_{n} \\
0 & \tau_{11} & \cdots & \tau_{1 n} \\
\cdots & \cdots & \cdots & \cdots \\
0 & \tau_{n 1} & \cdots & \tau_{n n}
\end{array}\right) \\
\pi_{i j}=-\pi_{j i}
\end{gathered}
$$

By the relation

$$
d(S T)(S T)^{-1}=d S S^{-1}+S\left(d T T^{-1}\right) S^{-1}
$$

we have

$$
\begin{aligned}
& \pi_{i}=\omega_{i}+\sum_{j} p_{i j}\left(\tau_{j}+\sum_{k} x_{k} \tau_{k j}\right) \\
& \pi_{i j}=\omega_{i j}+\sum_{k h} p_{i k} p_{j h} \tau_{k h} .
\end{aligned}
$$

(a) Euclidean spaces with points as its elements

In this case a group $G$ in the general theory is a group of displacement and $H$ is a group $S O(n)$. Principal relative components of our spaces $M=G / H$ are $\omega_{1}, \ldots, \omega_{n}$. We take a hypersurface $F$ to whose points we attach frames $S R$ of $n$-1-parameters. As a volume element of a part generated by a 1 parametric motion $T$ of the hypersurface we have by the use of (3.11)

Putting

$$
d V=\left[\pi_{1} \cdots \pi_{n}\right] .
$$

$$
\begin{aligned}
& \Omega_{i}=\sum_{j}(-1)^{i-1} p_{j i}\left[\omega_{1} \cdots \hat{\omega}_{j} \cdots \omega_{n}\right] \\
& \Omega_{i j}=x_{t} \Omega_{j}-x_{j} \Omega_{i}
\end{aligned}
$$

we have

$$
d V=\sum_{i}\left[\tau_{i} \Omega_{i}\right]+\sum_{i=j}\left[\tau_{i j} \Omega_{i j}\right]
$$

By the relation $\omega_{i}=\sum_{j} p_{i j} d x_{j}$ (3.13) reduces to

$$
\Omega_{i}=(-1)^{i-1}\left[d x_{1} \cdots \widehat{d x_{i}} \cdots d x_{n}\right] .
$$

When we put

$$
\begin{aligned}
& \Pi_{i}=\frac{1}{n-1} \sum_{j(\neq i)}(-1)^{i+j} x_{j}\left[d x_{1} \cdots \widehat{d x_{i}} \cdots \widehat{d x_{j}} \cdots d x_{n}\right] \\
& \Pi_{i j}=\frac{1}{2}\left(x_{i}^{2}+x_{j}^{2}\right)\left[d x_{1} \cdots \widehat{d x_{i}} \cdots \widehat{d x_{j}} \cdots d x_{n}\right]
\end{aligned}
$$

we have

$$
\Omega_{l}=d I I_{i}, \quad \Omega_{i j}=d I I_{i j}
$$

and $\Omega_{i}, \Omega_{i j}$ are forms on $M$, though they depend on a coordinate system.

If we take a domain $D$ on the hypersurface, an algebraic volume $V$ generated by a 1-parametric motion $T$ of $D$ is

$$
V=\sum_{i} X_{i} Y_{i}+\sum_{i=1} X_{i j} Y_{i j}
$$


where we have put $X_{i}=(-1)^{n-1} \int_{D} \Omega_{i}, X_{i j}=(-1)^{n-1} \int_{D} \Omega_{i j}, Y_{i}=\int \tau_{i}, Y_{i j}=\int \tau_{i j}$ $X_{i}, X_{i j}$ depend on the boundary of $D$ and not on $D$ itself. This is the theorem of Koenigs-Hadamard-Guillaumin.

If we take frames $\bar{S} R=S C R$ and a motion $\bar{T}=C^{-1} T$ with a constant matrix $C$, we get the same generation of $D$ and we have

$$
V=\sum_{i} X_{i} Y_{i}+\sum_{i<j} X_{i j} Y_{i j}
$$

and by (2.13), (3.8), (3.9) relations between $X_{i}, X_{i j}$ and $\bar{X}_{i}, \bar{X}_{i j}$ are

$$
\begin{aligned}
& \bar{X}_{i}=\sum_{j} X_{j} c_{j i} \\
& \bar{X}_{i j}=\sum_{k} X_{k}\left(c_{i} c_{k j}-c_{j} c_{k i}\right)+\sum_{k<h} X_{k h}\left(c_{k i} c_{h j}-c_{h i} c_{k j}\right) .
\end{aligned}
$$

By taking $\left(c_{i j}\right)$ suitably, we can assume

$$
\bar{X}_{2}=0, \ldots, \bar{X}_{n}=0 \text {. }
$$

For $c_{i j}=\delta_{i j}$ and $X_{2}=0, \ldots, X_{n}=0$ we have

$$
\bar{X}_{i 1}=c_{i} X_{1}+X_{i 1} \quad(i \neq 1) .
$$

Hence by taking $c_{i}$ suitably we can assume $\bar{X}_{i 1}=0$ in case $X_{1} \neq 0$. If frames are so chosen from first, we have

$$
V=X_{1} Y_{1}+\sum_{i<j} X_{i j} Y_{i j} \quad(i, j=2, \ldots, n)
$$

and by taking $\left(c_{i j}\right)$ suitably we can simplify in such a way that $X_{i j}(i<j)$ except $X_{23}, X_{45}, \ldots$ vanish. If $X_{1}=0$, we have

$$
V=\sum_{i<j} X_{i j} Y_{i j}
$$

where $X_{i j}(i<j)$ except $X_{12}, X_{34}, \ldots$ vanish.

Next we turn to an application of the Theorem 3. We take a hyperplane $E_{n-1}$ and denote by $J$ the set of all displacements which fix $E_{n-1}$. If we take a fundamental frame $\left(A^{\circ}, e_{1}^{\circ}, \ldots, e_{n}^{\circ}\right)$ with $A^{\circ}, e_{2}^{\circ}, \ldots, e_{n}^{\circ}$ on $E_{n-1}$, we have for a displacement $S$ of $J$ represented as in (3.3)

$$
x_{1}=0, \quad p_{11}=1, \quad p_{12}=0, \ldots, p_{1 n}=0 .
$$

Hence $\omega_{1}=0$ and by (3.13), (3.14)

$$
\Omega_{i}=p_{1 i}\left[\omega_{2} \cdots \omega_{n}\right], \quad \Omega_{i j}=x_{i} \Omega_{j}-x_{j} \Omega_{i}
$$

and so 


$$
\begin{aligned}
& \Omega_{1}=\left[\omega_{2} \ldots \omega_{n}\right], \quad \Omega_{i}=0 \quad(i \geqq 2) \\
& \Omega_{i 1}=x_{i} \Omega_{1}, \quad \Omega_{i j}=0 \quad(j>i \geqq 2) .
\end{aligned}
$$

For a domain $D(u)(0 \leqq u \leqq 1)$ on $E_{n-1}$ we take a suitable frame $S C R$ $(C \in J)$, then we have by (3.24) $\bar{X}_{i 1}=0$. Thus a volume generated by $D(u)$ under a motion $T=T(u)(0 \leqq u \leqq 1)$ for a suitably chosen frame on $D(u)$ is given by

$$
V=\int X_{1} \tau_{1}
$$

$X_{1}=\int_{D(u)}\left[\omega_{2} \cdots \omega_{n}\right]$ is an $n-1$-dimensional volume and $\tau_{1}$ is a component, orthogonal to the hyperplane on which $D(u)$ under $T$ lies, of an arcelement of the locus of center of gravitation of $D(u)$ (c.f. [7] p. 113).

We give another application of the Theorem 3. Let $K_{n-1}$ be a hypersphere of radius $r$ and $J$ be the set of all displacements which fix $K_{n-1}$. We take an origin $A^{\circ}$ of the fundamental frame at the center of $K_{n-1}$ and $\left(A, e_{1}, \ldots, e_{n}\right)$ with $A$ on $K_{n-1}$, and $e_{1}$ on an outer normal at $A$. Then we have $x_{i}=r p_{1 i}$ in (3.3). By the relation $d\left(A-r e_{1}\right)=\sum_{i} \omega_{i} e_{i}-r \sum_{i} \omega_{1 i} e_{i}=0$ we have along $K_{n-1}$

$$
\omega_{1}=0, \quad \omega_{i}-r \omega_{1 i}=0 \quad(i=2, \ldots, n) .
$$

Hence by (3.13), (3.14)

$$
\begin{aligned}
& \Omega_{i}=p_{1 i}\left[\omega_{2} \cdots \omega_{n}\right] \\
& \Omega_{i j}=x_{i} \Omega_{j}-x_{j} \Omega_{i}=r\left(p_{1 i} \Omega_{j}-p_{1 j} \Omega_{i}\right)=0 .
\end{aligned}
$$

We take a domain $D(u)$ on $K_{n-1}$ and an algebraic volume gemerated by $D(u)$ under a motion $T=T(u)(0 \leqq u \leqq 1)$ of $K_{n-1}$ is

$$
V=\sum_{i} \int X_{i} \tau_{i}, \quad \text { where } \quad X_{i}=(-1)^{n-1} \int_{D(u)} \Omega_{i}
$$

If $v$ is an $n$-1-dimensional volume of $D(u)$ and $k=(-1)^{n-1} r v^{-1},\left(k X_{1}, \ldots, k X_{n}\right)$ are coordinates of a center of gravitation of $D(u)$. By taking on each $D(u)$ a suitable frame we can reduce $X_{i}$ to such ones that $X_{2}=0, \ldots, X_{n}=0$. Hence

$$
V=\int X_{1} \tau_{1}
$$

Thus we get (3.32), where $V$ is an algebraic volume generated by a set of domains of $D(u)$ on a hypersphere $K_{n-1}$ of radius $r$ under a motion $T=T(u)$ $(0 \leqq u \leqq 1)$ and $r^{-1} v X_{1}$ is a distance from the center of $K_{n-1}$ to a center of 
gravitation $g(u)$ of $D(u)$, and $\tau_{1}$ is an orthogonal component of an arcelement of the locus of center of the hypersphere $K_{n-1}$ to the direction from the center of $K_{n-1}$ to $g(u)$. According to the general theory a formula

$$
V=\int X_{1} \tau_{1}+\sum_{i} \int\left(X_{i}-r X_{1 i}\right)\left(\tau_{i}-r \tau_{1 i}\right)
$$

should appear in the first on account of (3.30). A modification has been made by taking an origin $A^{\circ}$ at the center of $K_{n-1}$ from first.

(b) Euclidean space with hyperplanes as its elements

In this case we take frames $\left(A, \mathrm{e}_{1}, \ldots, e_{n}\right)$ with $A, e_{2}, \ldots, e_{n}$ on a hyperplane. Then principal relative components are $\omega_{1}, \omega_{12}, \ldots, \omega_{1 n}$. We take an $n-1$-parametric set $D$ of hyperplanes to which we attach an $n-1$ parametric set of frames $S R$ and a 1-parametric motion $T=T(u)(0 \leqq u \leqq 1)$. Then an element $d V$ of an algebraic volume generated by $D$ under a motion $T$ is given by

$$
d V=\left[\pi_{1} \pi_{12} \cdots \pi_{1 n}\right],
$$

where $\pi_{1}, \pi_{1 i}$ are given by (3.10). We put

and also

$$
\begin{gathered}
p_{i}=p_{1 i}, \quad h=\left(A, e_{1}\right), \quad d \sigma=\left[\omega_{12} \cdots \omega_{1 n}\right], \\
A_{i}=\sum_{k}(-1)^{k-1} p_{k i}\left[\omega_{12} \cdots \hat{\omega}_{1 k} \cdots \omega_{1 n}\right]
\end{gathered}
$$

$$
\begin{aligned}
& \Omega_{i}=p_{i} d \sigma \\
& \Omega_{i j}=x_{i} \Omega_{j}-x_{j} \Omega_{i}+p_{i}\left[\omega_{1} A_{j}\right]-p_{j}\left[\omega_{1} \Lambda_{i}\right] .
\end{aligned}
$$

Then we get by (3.11) (3.12)

$$
d V=\sum_{i}\left[\tau_{i} \Omega_{i}\right]+\sum_{i<j}\left[\tau_{i j} \Omega_{i j}\right] .
$$

$d_{\sigma}$ is an $n$-1-dimensional volume element of spherical representation of normals of hyperplanes belonging to $D$, and $h=\left(A, e_{1}\right)$ is a distance from the origin of a fundamental frame to a hyperplane. By the general theory $\Omega_{i}, \Omega_{i j}$ are closed forms, which can also be verified directly. As for $\Omega_{i j}$ we have

$$
\Omega_{i j}=\left[d h, p_{i} \Lambda_{j}-p_{j} \Lambda_{i}\right]
$$

because of the relations

$$
d h=d\left(A, e_{1}\right)=\left(d A, e_{1}\right)+\left(A, d e_{1}\right)=\omega_{1}+\sum_{i j} \omega_{1 i} p_{i j} x_{j},
$$

and $\Omega_{i j}$ are closed forms on account of the formula 


$$
\Lambda_{i}=(-1)^{i} \sum_{j}(-1)^{j-1} p_{j}\left[d p_{i} \cdots \widehat{d p}_{i} \cdots \widehat{d p}_{j} \cdots d p_{n}\right]
$$

An algebraic volume $V$ generated by a domain $D$ of hyperplanes under a 1 parametric motion $T$ is

$$
V=\sum_{i} X_{i} Y_{i}+\sum_{i<j} X_{i j} Y_{i j}
$$

where $X_{i}, X_{i j}, Y_{i}, Y_{i j}$ are as in (3.20) and a simplification of (3.39) are quite the same as in $(3.25),(3.26)$. We take a set of all hyperplanes through one point and a rotation group $S O(n)$ around the point. Then for frames $S R$ $(S \in S O(n))$ with an origin $A$ at the point we have

$$
x_{1}=0, \ldots, x_{n}=0, \quad \omega_{1}=0, \cdots, \omega_{n}=0
$$

and for these frames $\Omega_{i j}$ in (3.35) vanish and $X_{i j}=0$. Hence an algebraic volume $V$ of a set $D(u)$ of hyperplanes passing through $A=A(u)(0 \leqq u \leqq 1)$ is given by

$$
V=\sum_{i} \int X_{i} \tau_{i}, \quad \text { where } \quad X_{i}=(-1)^{n-1} \int_{D(u)} \Omega_{i}
$$

We take a spherical representation of normals of hyperplanes, and the center of gravitation of the representation of $D(u)$ has coordinates $\left(k X_{1}, \ldots, k X_{n}\right)$, $v$ being an $n$-1-dimensional volume of the spherical representation, and $k=(-1)^{n-1} v^{-1}$. By a assuitable choice of $C$ for which $c_{i}=0$ we have

$$
\bar{X}_{i}=\sum_{j} X_{j} c_{j i}
$$

by (3.22) and by a suitable choice of $\left(c_{i j}\right)$ we get a formula

$$
V=\int X_{1} \tau_{1}
$$

where $v^{-1} X_{1}$ is a distance from $A(u)$ to a center of gravitation $g(u)$ of $D(u)$ and $\tau_{1}$ is a component of an arcelement of the locus of a center $A(u)$ to the direction from $A(u)$ to $g(u)$.

(c) Euclidean space with straight lines as its elements

We take an origin $A$ and $e_{1}$ of a frame on a straight line. Then principal relative components of the space with straight lines as its elements are

$$
\omega_{2}, \ldots, \omega_{n}, \quad \omega_{12}, \ldots, \omega_{1 n} .
$$

For an element $d V$ of an algebraic volume generated by a $2 n-3$-parametric set $D$ of straight lines under a 1-parametric motion we have 


$$
d V=\left[\pi_{2} \cdots \pi_{n}, \pi_{12} \cdots \pi_{1 n}\right]
$$

in the notation as in (3.10). We put

and also

$$
\begin{gathered}
p_{i}=p_{1 i}, \quad d S=\left[\omega_{2} \cdots \omega_{n}\right], \quad d \sigma=\left[\omega_{12} \cdots \omega_{1 n}\right], \\
M_{i}=\sum_{i}(-1)^{j-1} p_{j i}\left[\omega_{2} \cdots \hat{\omega}_{j} \cdots \omega_{n}\right] \\
\Lambda_{i}=\sum_{j}(-1)^{j-1} p_{j i}\left[\omega_{12} \cdots \hat{\omega}_{1 j} \cdots \omega_{1 n}\right]
\end{gathered}
$$

$$
\begin{aligned}
& \Omega_{i}=\left[M_{i} d \sigma\right] \\
& \Omega_{i j}=x_{i} \Omega_{j}-x_{j} \Omega_{i}+(-1)^{n}\left[d \mathrm{~S}, p_{i} \Lambda_{j}-p_{j} \Lambda_{i}\right] .
\end{aligned}
$$

These are closed forms by the general theory although they are also verified by

$$
M_{i}=(-1)^{i} \sum_{j}(-1)^{j-1} p_{j}\left[d x_{1} \cdots \widehat{d x}_{i} \cdots \widehat{d x}_{j} \cdots d x_{n}\right]
$$

and (3.38). An algebraic volume generated by a set of straight lines under a 1-parametric motion is given by (3.19).

If we consider a set of all straight lines which are perpendicular to a fixed direction which we take as $e_{n}$, we have

$$
p_{1 n}=0, \ldots, p_{n-1 n}=0, \quad p_{n n}=1, \quad \omega_{1 n}=0, \ldots, \omega_{n-1 n}=0
$$

and so by virtue of (3.43) (3.44) we get

$$
\Omega_{i}=0, \quad \Omega_{i j}=0 \quad(i<j<n) .
$$

We consider a $2 n-3$-parametric set $D(u)$ of straight lines which are perpendicular to a fixed direction each. An algebraic volume $V$ generated by a set $D(u)(0 \leqq u \leqq 1)$ is given by

$$
V=\sum_{i} \int X_{i n} \tau_{i n}
$$

A transformation $C$ which fixes a direction $e_{n}$ satisfies the relation $c_{1 n}=0, \ldots$, $c_{n-1 n}=0, c_{n n}=1$ and by (3.23) we can reduce $X_{1 n}, \ldots, X_{n-1 n}$ to $X_{i n}=0(i \geqq 2)$, and we get a formula

$$
V=\int X_{1 n} \tau_{1 n}
$$

\section{BIBLIOGRAPHY}

[1] G. Koenigs, Sur la détermination générale du volume engendré par un contour fermé. gauche ou plan dans un mouvement quelconque, Jour. de Math. 1889. 
[2] J. Hadamard, Sur la généralization du théorème de Guldin, Bull. des Sc. Math. t. 26, 1898.

[3] A. Bloch and G. Guillaumin, La géométrie integrale du contour gauche, Gauthier Villars, 1949.

[4] E. Cartan, La théorie des groupes finis et contunus et la géométrie différentielle par la methode du reperes mobiles, Gauthier Villars, 1938.

[5] W. Blaschke, Integralgeometrie, Actualites scientifiques et industrielles, 1935.

[6] M. Kurita, On the vector in homogeneous spaces, Nagoya Math. Jour. vol. 5, 1953.

[7] M. Kurita, On some formulas about volume and surface area, Nagoya Math. Jour. vol.6, 1954.

Mathematical Institute

Nagoya University 